Check for updates

Cite this: RSC Adv., 2019, 9, 1541

Received 12th December 2018

Accepted 8th January 2019

DOI: $10.1039 / \mathrm{c} 8 \mathrm{ra10200g}$

rsc.li/rsc-advances

\section{Sintered porous Ti6Al4V scaffolds incorporated with recombinant human bone morphogenetic protein-2 microspheres and thermosensitive hydrogels can enhance bone regeneration}

Ji Li, (D a ${ }^{\text {a }}$ Zhongli Li, ${ }^{\text {a a }}$ Qi Wang, ${ }^{\text {b }}$ Yueyi Shi, ${ }^{c}$ Wei Li, ${ }^{a}$ Yangmu Fu ${ }^{a}$ and Gong Jin ${ }^{d}$

A well-controlled powder sintering technique was used to fabricate porous Ti6Al4V scaffold. The thermosensitive chitosan thioglycolic acid (CS-TA) hydrogel was used as a carrier to inject recombinant human bone morphogenetic protein-2 (rhBMP-2) microspheres into pores of the Ti6Al4V scaffold at $37{ }^{\circ} \mathrm{C}$, and then the porous Ti6Al4V/rhBMP-2 loaded hydrogel composite was obtained. The bare Ti6Al4V scaffold was used as the control. The characteristics and mechanical properties of the scaffold, rheological properties of the hydrogels and the rhBMP-2 loaded hydrogel, the release of the rhBMP-2 loaded hydrogel, and the biological properties of the two types of samples were evaluated by in vitro and in vivo tests. Results indicated that the sintered porous Ti6Al4V had high porosity, large pore size with good mechanical properties. The hydrogel and rhBMP-2 loaded hydrogel showed thermosensity. The rhBMP-2 loaded hydrogel showed a stable and extended release profile without too high burst release of rhBMP-2. Both groups showed good biocompatibility and osteogenic ability. However, according to the results of cell tests and implantation, the group with rhBMP-2 loaded hydrogel had significantly higher cell proliferation rate, faster bone growth speed, and more bone ingrowth at every time point. Therefore, the sintered porous Ti6Al4V scaffolds incorporated with rhBMP-2 microspheres and CS-TA hydrogel was effective in enhancing the bone regeneration, and prospects a good candidate for application in orthopedics.

\section{Introduction}

Various research studies have been conducted for developing materials for the regeneration of bone defects and fracture repair, which remains a challenging topic in orthopedic and maxillofacial surgery. ${ }^{1,2}$ But most of them have shown very limited capability. ${ }^{3}$ Recently, porous Ti6Al4V has attracted enormous interest because of its excellent properties. ${ }^{4} \mathrm{~A}$ certain degree of porosity allows reducing the elastic modulus of Ti6Al4V and the stiffness mismatch between bone and the Ti6Al4V implant, and hence decrease the potential risks of stress shielding on bone resorption. ${ }^{5,6}$ The porous structure provides the necessary space for cell and bone grow in with sufficient mechanical support ${ }^{7,8}$ to mimic the properties of the

aDepartment of Orthopedics, General Hospital of PLA, No. 28 Fuxing Road, Haidian District, Beijing 100853, China. E-mail: lizhongli@263.net; Fax: +86 010 66938306; Tel: +8601066938306

${ }^{b}$ Department of Orthopedics, Characteristic Medical Center of PAP, Tianjin 300162, China

'Department of Stomatology, General Hospital of PLA, No. 28 Fuxing Road, Haidian District, Beijing 100853, China


Technological Development Zone, Beijing 100176, China extracellular matrix. ${ }^{9}$ However, porous Ti6Al4V scaffolds remain bioinert and thereby lack the ability to provide strong biological cues that can enhance the regeneration of bone. ${ }^{7}$ Recently, many research studies in regenerative medicine have used various biologic factors to further improve the biologic activity of the bone graft substitutes. ${ }^{10}$

Bone morphogenetic protein (BMP) is a kind of growth factor that promotes bone and cartilage formation. Recombinant human BMP-2 (rhBMP-2) has been purified ${ }^{11}$ and shown to enhance in vivo bone formation. ${ }^{12}$ BMP has been used for decades in treating spinal fusion, dental implantation, and implant osseointegration with excellent results. ${ }^{13}$ McClellan et al. noted that rhBMP-2 resulted in a quicker, more robust fusion. ${ }^{14}$ Clinically, however, BMP-2 promotion of bone formation is markedly limited due to its rapid diffusion and absorption when it is injected into the body. ${ }^{15}$ It is reported that sustained growth factor delivery can be established through different non-covalent retention mechanisms, ${ }^{16}$ including physical entrapment, absorption, and complexation. ${ }^{17}$ Entrapping BMP in the microspheres can prolong the sustained release of BMP-2, maximizing its ability to induce bone formation. Our previous study indicated that sustained release (over 1 month) of rhBMP-2 were achieved from rhBMP-2-loaded poly(- 
lactic-co-glycolic acid) (PLGA) microspheres. ${ }^{18}$ However, rhBMP2 microspheres were reported to have an initial burst release in vitro. ${ }^{12}$ High doses of growth factor, especially BMP-2, can cause serious side effects, including bone tissue overgrowth, ectopic bone formation, inflammation, and even carcinogenicity. ${ }^{19}$ The previous study reported that hydrogels with a high viscosity can provide high control of the release growth factors. ${ }^{7,20}$ However, the hydrogels lack sufficient mechanical properties necessary for load-bearing applications. Therefore, when rhBMP-loaded hydrogel are incorporated into porous Ti6Al4V scaffolds this may result in a biologically active and mechanically strong composite biomaterial.

In the present work, a highly controlled powder sintering technique was used for the fabrication of porous Ti6Al4V scaffold with high porosity and large pores. A modified thermosensitive chitosan hydrogel was used as the carrier to translate the growth factor into the pores of the Ti6Al4V scaffold. This type of hydrogels could remain liquid at low temperatures and become a gel at $37{ }^{\circ} \mathrm{C}$ or in the human body, which is very important for the control of the release of BMP- 2 and is very convenient for the use in clinic. We hypothesize that the rhBMP2 loaded thermosensitive hydrogels can enhance the biological activity and osteogenesis ability of Ti6Al4V scaffold, and in vitro and in vivo experiments were performed.

\section{Materials and methods}

\subsection{Preparation of porous Ti6Al4V scaffold}

Ti6Al4V powder with particle size $<100 \mu \mathrm{m}$ was used for the fabrication in this technique. The scaffolds were produced as previously described. ${ }^{21-23}$ In brief, the Ti6Al4V powder mixed with $\mathrm{H}_{2} \mathrm{O}$, agar, Tergitol TMN, triton and ammonium alginate were used to prepare a powder suspension. It was mixed for $6 \mathrm{~min}$ at $70{ }^{\circ} \mathrm{C}$ to obtain a fluid foam. The foam is cast into a mould and cooled down until the structure is gelled. After demoulding, the structure is dried at atmospheric pressure and then the green artifact was calcined with a slow heating step at a rate lower than or equal to $20{ }^{\circ} \mathrm{C}$ per hour to a temperature between 400 and $600{ }^{\circ} \mathrm{C}$ under an inert atmosphere (Ar) or at a pressure more than $10^{-3}$ mbar and constant temperature for $2 \mathrm{~h}$. Then, the artifact was sintered with slow heating to a temperature of $1250{ }^{\circ} \mathrm{C}$ under vacuum or at a pressure more than $10^{-4}$ mbar and constant temperature for $2 \mathrm{~h}$.

Three types of porous Ti6Al4V samples were prepared in this study: rod specimens of $12 \mathrm{~mm}$ in diameter and $22 \mathrm{~mm}$ in length were used for the mechanical tests; disk specimens of $3 \mathrm{~mm}$ thick and $10 \mathrm{~mm}$ diameter were used for in vitro biochemical tests; rod specimens of $2 \mathrm{~mm}$ in diameter and $6 \mathrm{~mm}$ in length were used for the in vivo animal experiments. The samples were ultrasonically treated in order to remove impurities. And then the samples were sealed in sterile bags and steam autoclaved for $15 \mathrm{~min}$ at $120^{\circ} \mathrm{C}$.

\subsection{Characterizations of the porous Ti6Al4V scaffold}

Although characterizations of the porous sample, such as pore size and porosity, have been determined in our previous study, ${ }^{22,23}$ the slight differences in different batches still existed. Thus, in order to ensure the accuracy of the study and the success fabrication of the porous Ti6Al4V, the samples were characterized. An optical microscope was used to examine the surface topography and microstructure of the porous Ti6Al4V scaffold. The mechanical integrity of each cell strut was observed by a micro-CT. A scanning electron microscopy (SEM) (Hitachi FE-SEM SU3500, Japan) was used to qualitatively determine the microtopography (such as the pore size). Ten values were measured in each vision and then the average pore diameter value was calculated. Porosity was calculated by bulk density and apparent density. The stiffness of the porous Ti6Al4V sample was evaluated by an axial compression test using an MTS Alliance RT30 electromechanical test frame (MTS systems, USA) as previous described. ${ }^{22,23}$ In brief, a compressive test to failure was done at a crosshead speed of $0.5 \mathrm{~mm} \mathrm{~min} \mathrm{~m}^{-1}$ and the maximum load of the machine was $20000 \mathrm{~N}$, and the real-time load/displacement was recorded. The stress-strain curves were made and then the elastic modulus was calculated for each sample.

\subsection{Preparation and characterizations of rhBMP-2 microspheres}

A previously described water-in-oil in water $(\mathrm{W} / \mathrm{O} / \mathrm{W})$ doubleemulsion-solvent-extraction technique was used to fabricate microspheres. ${ }^{18,24}$ Initially, $50 \mu \mathrm{g}$ solid rhBMP-2 was homogenized with $150 \mu \mathrm{l}$ phosphate buffered saline (PBS), which serves as the inner water phase. Then $400 \mathrm{mg}$ poly(lactic-co-glycolic acid) (PLGA) was dissolved in $1 \mathrm{ml}$ of dichloromethane as the oil phase. An emulsion was formed by homogenization for $30 \mathrm{~s}$ with an ultrasound in an ice bath and then dropped into $65 \mathrm{ml}$ of ice-cooled 1\% polyvinyl alcohol (PVA), a vortex at $5600 \mathrm{rpm}$ was used to create the double emulsion and, subsequently, the microspheres. The dichloromethane was evaporated for $3 \mathrm{~h}$ with magnetic stirring at low speed. The microspheres were collected by centrifugation, washed three times with distilled deionized water, and vacuum-dried to obtain powder. The rhBMP-2 microspheres power were stored at $4{ }^{\circ} \mathrm{C} .{ }^{18}$

The outer phase of the $\mathrm{W} / \mathrm{O} / \mathrm{W}$ emulsion and the washing water were assessed for encapsulation efficiency. The amount of rhBMP-2 in the washing water was used to determine by rhBMP2 ELISA kit (Sigma, USA). The amount of rhBMP-2 in the microspheres was calculated by subtracting the rhBMP-2 in the washing water $(W)$ from the total amount of rhBMP-2 $(T)$ used during fabrication, and then the encapsulation efficiency was calculated: $E=[(T-W) / T] \% .^{18}$

\subsection{Preparation of the thermosensitive hydrogels}

Initially, $40 \mathrm{mg}$ chitosan thioglycolic acid (CS-TA), $960 \mu \mathrm{l} \beta$ sodium glycerophosphate $\left(0.8 \mathrm{mmol} \mathrm{l}^{-1}\right)$ and $10 \mu \mathrm{l}$ poly(ethylene glycol) diacrylate (PEGDA) was used as the starting material. The mixture was homogenized with ultrasound and stirred using a vortex. The resulting fluid CS-TA hydrogel was stored at $4{ }^{\circ} \mathrm{C}$. Then the temperature sensitivity of the CS-TA hydrogel was evaluated by placing the hydrogels in a $37{ }^{\circ} \mathrm{C}$ 




Fig. 1 Schematic illustration of the preparation of the porous Ti6Al4V and rhBMP-2 loaded hydrogel system.

water bath. The time required for the hydrogel consistency to change from a fluid to a gel was recorded.

\subsection{Rheological properties of the hydrogels and the rhBMP-2 loaded hydrogel}

The Physica MCR101 rheometer (Antonpaar Ltd, Austria) was used to measure the rheological properties of the thermosensitive hydrogels and rhBMP-2 loaded hydrogels. The strain was $1 \%$, the frequency was $1 \mathrm{~Hz}$, the scanning temperature range was $4-50{ }^{\circ} \mathrm{C}$, and the heating rate was $1{ }^{\circ} \mathrm{C} \mathrm{min}^{-1}$. A constant temperature water bath controls the temperature of the measurement system. $3 \mathrm{ml}$ CS-TA hydrogel and $3 \mathrm{ml} \mathrm{rhBMP-}$ 2 loaded hydrogel were used for the measurement. The surface is covered with dimethicone to prevent evaporation. Dimethicone has no effect on the gelation temperature of the hydrogel. The values of storage modulus $\left(G^{\prime}\right)$ and loss modulus $\left(G^{\prime \prime}\right)$ were recorded and then the curve of $G^{\prime}$ and $G^{\prime \prime}$ with temperatures were generated. The temperature where the values of $G^{\prime}$ equalled with $G^{\prime \prime}$ was defined as gelation temperatures.

\subsection{In vitro release of rhBMP-2 microspheres and the rhBMP-2 loaded hydrogel}

Eighty milligrams of rhBMP-2 microspheres was added with $2.4 \mathrm{ml}$ PBS and $2.4 \mathrm{ml} \mathrm{CS}$-TA hydrogel for the test. Eighty $\mathrm{mg}$ of the microspheres mixed with $4.8 \mathrm{ml}$ PBS were as the control. The two rhBMP-2 sustained-release systems were homogenized and divided into $240.1 \mathrm{ml}$ Eppendorf tubes, which were then diluted with PBS buffer to a final volume of $1 \mathrm{ml}$. Each sample was placed in a constant temperature shaker (maintained at $\left.37^{\circ} \mathrm{C}, 100 \mathrm{rpm}\right)$ and analyzed at each time point $(8 \mathrm{~h}, 1 \mathrm{~d}, 2 \mathrm{~d}, 3$ d, $7 \mathrm{~d}, 14 \mathrm{~d}$, and $21 \mathrm{~d}) .{ }^{18}$ A sandwich ELISA kit (Sigma, USA) was used to determine the concentration of rhBMP-2. All batches were assessed in triplicate.

\subsection{Preparation of the composite}

We used $5 \mathrm{mg}$ of rhBMP-2 microspheres combined with liquid CS-TA at $4{ }^{\circ} \mathrm{C}$, and the rhBMP-2 microspheres were embedded into the CS-TA hydrogel. A total of 24 porous Ti6Al4V disc samples were coated with the rhBMP-2 microsphere-loaded CS-
TA hydrogel for the in vitro biochemical assays. A total of 24 porous Ti6Al4V rod samples were coated with the rhBMP-2 microsphere-loaded CS-TA hydrogel for the in vivo implantations. The samples for implantation were prepared before being inserted in the animal. The liquid rhBMP2-loaded CS-TA would gel when concurrently injected into the scaffolds' porous space in a $37{ }^{\circ} \mathrm{C}$ water bath. The schematic illustration of the preparation of the composite was shown in the Fig. 1.

\subsection{In vitro biochemical assays}

2.8.1 Cell proliferation and attachment. Continuous cell lines of human osteoblasts (OB) (MG-63) were used for the cell tests. Cells were cultured in DMEM supplemented with $10 \%$ BCS and $1 \%$ penicillin/streptomycin and incubated at $37{ }^{\circ} \mathrm{C}$ in a $5 \% \mathrm{CO}_{2}, 100 \%$ relative humidity incubator. A third passage cell suspension $\left(2.5 \times 10^{5}\right.$ cells per $\left.\mathrm{ml}, 40 \mu \mathrm{l}\right)$ was placed in the sample in a 48-well plate and cultured. The medium was then added, and the cells were incubated with changing medium every 2-3 days. The cell viability was assessed using the live/ dead cell viability kit after culturing the cells for 1,3 and 7 days. Fluorescence images were obtained by positive fluorescence microscopy. Cell viability and proliferation was quantified by the Cell Counting Kit-8 assay (CCK-8) after culturing for 1,3 , and 7 days. The morphologies of cells attached on the material were observed after culturing the cells for 7 days with the two type of materials, the samples were rinsed with phosphate buffered saline to remove nonadherent cells and then fixed with $2.5 \mathrm{wt} \%$ glutaraldehyde for $1 \mathrm{~h}$. A series of gradient ethanol solutions (50-100\%) was used for sample dehydration, which was followed by the addition of pure isopentyl acetate. After being sputter-coated with a $10 \mathrm{~nm}$ thick gold film, cell morphology was observed by SEM (Hitachi FE-SEM S3500, Japan). The methods to evaluate the cell proliferation and attachment were according to the published studies. ${ }^{22,23}$

2.8.2 Cell differentiation. The bare porous Ti6Al4V scaffold and Ti6Al4V/rhBMP-2-loaded CS-TA hydrogel constructs were incubated in 48-well cell culture dishes with growth medium. After being cultured for 7 and 14 days, the cells were harvested. Total RNA was extracted and reverse transcribed to cDNA and 
then the expression levels of alkaline phosphatase (ALP), bone sialoprotein (BSP), Runx-2, collagen type I (COL I) and osteocalcin were quantified using the RT-PCR instrument.

\subsection{Implantation}

2.9.1 Animals and surgical procedure. Forty-eight New Zealand white rabbits (aged 4-6 months and weighing $3.5 \pm 0.3$ $\mathrm{kg}$ ) were obtained from the zoological animal center of our hospital, and 24 were in each group. All surgical procedures were performed in accordance with the ARRIVE guidelines and approved by the IRB, the detailed operations were described in our previous study. ${ }^{23}$ The surgeries were performed under standard sterile conditions. Firstly, the rabbits were anesthetized with an intramuscular injection of a $1: 1$ mixture of ketamine hydrochloride and xylazine hydrochloride, and both of the hind limbs were clipped and prepared for surgeries. A straight $2 \mathrm{~cm}$ skin incision was made over the portion of femoral, and $2 \mathrm{~mm}$ diameter drills were used to create cylindrical defects in the diaphysis of each femur, and then the implant was inserted. After implantation, the surrounding muscles were repositioned, and the subcutaneous tissues and the skin incision were repaired with resorbable sutures. The bare porous Ti6Al4V specimens and the Ti6Al4V/BMP-2-loaded CS-TA hydrogel constructs were implanted into the limbs of the rabbits. The animals received a single intramuscular injection of clavulanate-potentiated amoxicillin at the time of surgery and were then inspected daily for clinical signs of complications or adverse reactions. The rabbits were allowed unrestricted cage activity postoperatively.

2.9.2 Histological and histomorphometric analysis. To compare the rates of bone ingrowth in the two groups, sequential fluorochrome markers were used. The detailed operations were similar to our previous study. ${ }^{23}$ Briefly, all rabbits were intramuscular injected of tetracycline $\left(10 \mathrm{mg} \mathrm{ml}^{-1}\right.$, Sigma, USA) with $30 \mathrm{mg} \mathrm{kg}^{-1}$ and calcein green $\left(10 \mathrm{mg} \mathrm{ml}^{-1}\right.$, Sigma, USA) with $10 \mathrm{mg} \mathrm{kg}^{-1}$ at 2 weeks and 3 days before they were sacrificed, respectively. The rabbits were sacrificed by intra-cardiac overdose of anesthetic solution at 1, 4 and 8 weeks after implantation (eight rabbits at each time point). All retrieved implant-containing bone fragments were fixed in $70 \%$ ethanol for 2 weeks, and then dehydrated in a graded ethanol series $(70 \%$ to $100 \%)$. They were then embedded in a methyl methacrylate solution for polymerization at $37{ }^{\circ} \mathrm{C}$ for 1 week without decalcification. Then samples were subsequently cut and ground into $30 \mu \mathrm{m}$ transverse sections slices using a modified interlocked diamond saw (Leica Microtome, Wetzlar, Germany). After that, a laser confocal microscope (Leica, Germany) was used to observed the fluorochrome markers. The bone mineralization apposition rate (MAR, the vertical spacing between two fluorochrome markers/injection interval) was analyzed from the images of fluorochrome labeling, which generally indicated the new bone growth rate. After the fluorescence analysis, the samples were stained with hematoxylin and eosin (H\&E). Qualitative microscopic analyses were performed using an optical microscope attached to a digital camera. The bone-implant contact (BIC) values were determined in both groups by Image J software (Microsoft, Maryland, USA). Hemorrhage, necrosis, exudation, neovascularization cell, and encapsulation in the implant sites were evaluated macroscopically in a semi-quantitative manner.

\subsection{Statistical analysis}

The data were analyzed by Statistical Package for Social Sciences 17.0 software (SPSS 17.0, USA), the graphs were drawn by SPSS or GraphPad software (USA), and the results are presented as the mean \pm standard deviation (S.D). Statistical differences were evaluated by one-way ANOVA or Student's $t$-test. $P<0.05$ indicated a statistically significant difference.

\section{Results}

\subsection{Characterizations of the porous Ti6Al4V scaffold}

Surface of the porous Ti6Al4V scaffold was rough, but the struts were well formed and continuous (Fig. 2a). Pore morphology and distribution were demonstrated by SEM (Fig. 2b). There were amount of pores with different sizes and shapes, the $3 \mathrm{D}$ connectivity were observed between them. The porous structure may allow more rhBMP-2 microspheres to adhere to the


Fig. 2 Characterization of the sintered porous Ti6Al4V scaffold: (a) is cross-section images obtained by micro-CT, the scale bar represents 500 $\mu \mathrm{m}$; (b) is the SEM image, and scale bar represents $1 \mathrm{~mm}$. 
a



b

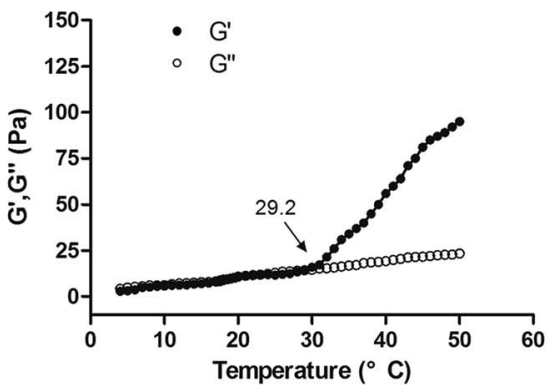

C

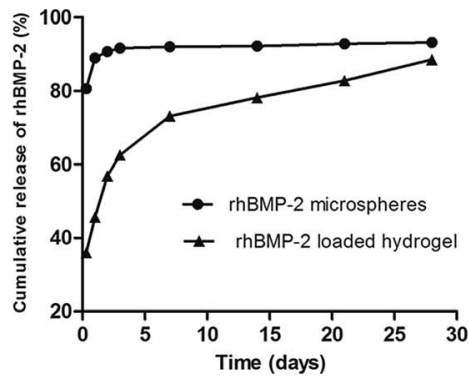

Fig. 3 (a) Temperature dependence of storage modulus $G^{\prime}$ and loss modulus $G^{\prime \prime}$ of the CS-TA hydrogel; (b) temperature dependence of storage modulus $G^{\prime}$ and loss modulus $G^{\prime \prime}$ of the rhBMP-2 loaded hydrogel; (c) in vitro release of rhBMP-2 microspheres and the rhBMP-2 loaded hydrogel.

implant. Detailed observation of the SEM images confirmed the mechanical integrity of cell strut. After measurement and calculation, the average porosity of the scaffold sample was about $(74.8 \pm 1.76) \%$, and the average pore size was approximately $502.91 \pm 203.05 \mu \mathrm{m}$. After the mechanical tests, the compressive stress/strain values were calculated according to the force-displacement data. The elastic modulus was obtained from the slope of the compressive stress-strain curve in the linear elastic region. After calculation, the average compressive elastic modulus was about $1.83 \pm 0.27 \mathrm{GPa}$, and strength was about $97.52 \pm 6.51 \mathrm{MPa}$. These results were similar to our previous studies. ${ }^{22,23}$

\subsection{Encapsulation efficiency of rhBMP-2 microspheres}

One advantage of double emulsion techniques is the high encapsulation efficiency into PLGA. ${ }^{25}$ In this study, after calculation, the encapsulation efficiency was as high as $98.37 \% \pm$ 3.26. The high efficiency prevented reduced waste rhBMP-2.

\subsection{The thermosensitivity of hydrogel}

The hydrogel can flow and molded when initially constituted, but after heating in a $37^{\circ} \mathrm{C}$ water bath in about $2.62 \pm 0.87 \mathrm{~min}$, the hydrogel changed from mobile fluid to solidified gels. This process is often called "curing" and involves the formation of covalent crosslinks between polymer chains to form a macromolecular network. ${ }^{26}$

Rheological properties of hydrogel and the rhBMP-2 loaded hydrogel were showed in Fig. 3a and b. Values of the $G^{\prime}$ and $G^{\prime \prime}$ in both groups were low at the low temperature and increased with the temperature, but the $G^{\prime \prime}$ values showed higher increased rate. For the thermosensitive hydrogel alone, the curves of $G^{\prime}$ and $G^{\prime \prime}$ were cross at the temperature of $28.3^{\circ} \mathrm{C}$, indicating the gelation temperature was $28.3^{\circ} \mathrm{C}$; for the rhBMP2 loaded hydrogel, the curves of $G^{\prime}$ and $G^{\prime \prime}$ were cross at the temperature of $29.2^{\circ} \mathrm{C}$, indicating the gelation temperature was $29 .{ }^{\circ} \mathrm{C}$. $G^{\prime \prime}$ increased rapidly after the cross point in both groups, indicating the gelation process of the systems. The adding of the rhBMP-2 microspheres showed a little effects of the rheological properties.

\subsection{In vitro release of rhBMP-2}

In vitro release profiles were assessed for a period of 28 days. As shown in Fig. 3c, the burst release of the CS-TA hydrogel release system was approximately $36 \%$ within the first 8 hours, compared with approximately $80 \%$ for the rhBMP-2 microspheres alone. In the 28 days test, the rhBMP-2 loaded hydrogel showed a stable and extended release profile. This may be due to the high viscosity of the CS-TA hydrogel.

\subsection{In vitro biochemical assays}

3.5.1 Cell proliferation and attachment. Fluorescence images of the live/dead assay were shown in Fig. 4, a homogenous distribution of living cells (green) on the sample surfaces with relatively few dead cells (red) were observed at every time point. All cells in each group showed good viability. The fluorescence images at 1,3 and 7 days showed that cell proliferation increased with time, and significant more cells in the Ti6Al4V/ hydrogel group were observed than the bare group (Fig. 4a and c). SEM images showed that the cells proliferated and attached well on the surface of porous Ti6Al4V in both groups, whereas the cells proliferated significantly faster and highly concentrated on the surface of the scaffold with rhBMP-2loaded hydrogel. Even more, OB cells grown from the edge into the pores and interconnected confluently with the presence of rhBMP-2 loaded hydrogel (Fig. 4b and d). In the CCK-8 results, both groups showed an increase of the optical density (OD) values with time, significant differences between each time quantum in each group were observed $(P<0.05)$. However, the group with rhBMP-2 loaded hydrogel showed significantly higher values than the bare group at every time point (Fig. 5 CCK-8). These results suggest that the porous Ti6Al4V and rhBMP-2 have no cytotoxic effect on OBs, and the rhBMP-2 loaded hydrogel may significantly promote the cells growth.

3.5.2 Osteogenic differentiation. After induction in normal growth medium for 7 and 14 days without the addition of osteogenic agents such as ascorbic acid, dexamethasone, and $\beta$ glycerophosphate, the expression of osteogenic associated proteins and marker genes was detected by qRT-PCR. Fig. 5 showed that, in both groups, the quantitative assessments showed a significant increase with time. The quantitative 

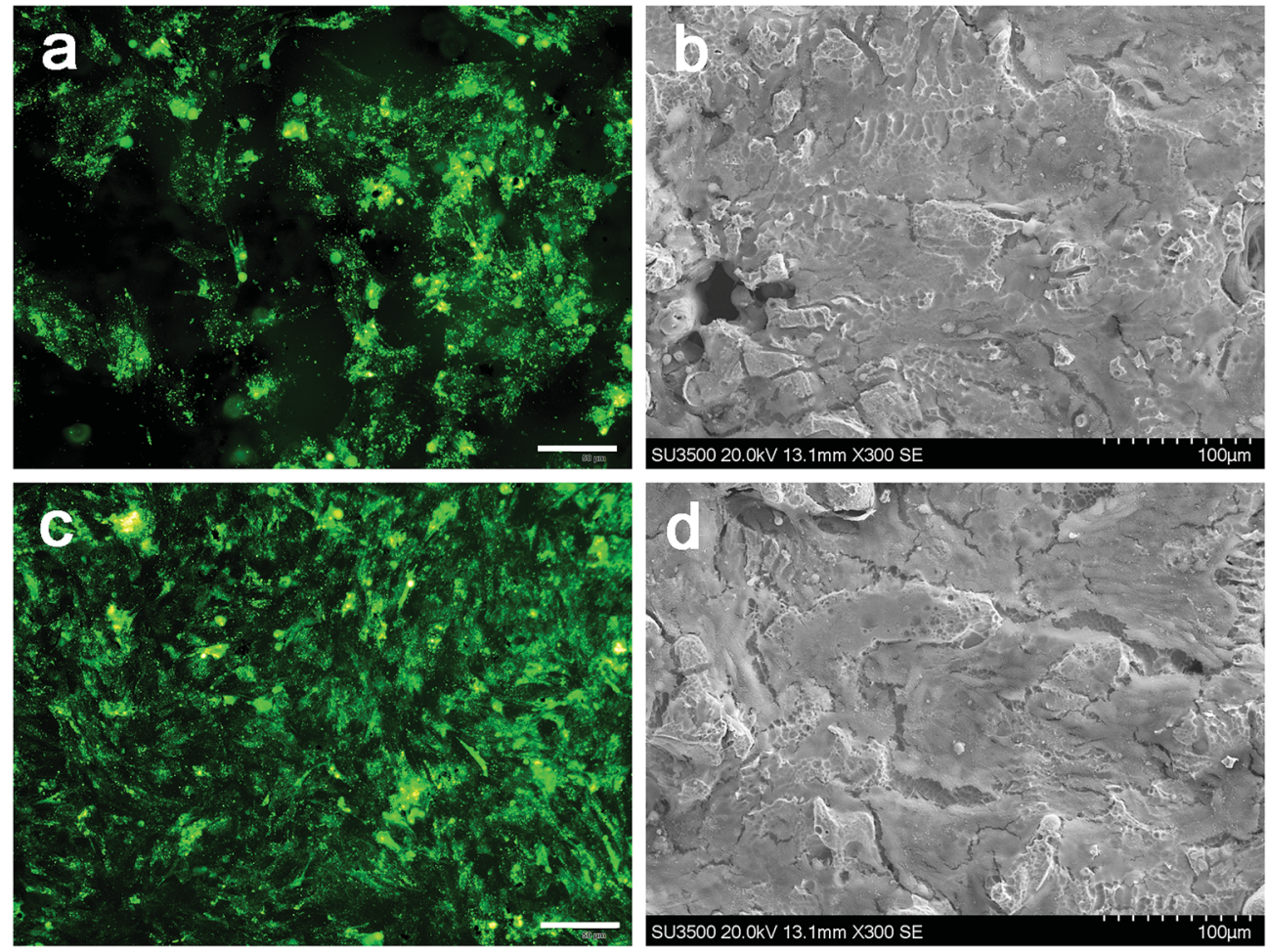

Fig. 4 Cell proliferation and live/dead fluorescence microscopy images after of the two groups: (a) bare Ti6Al4V scaffold and (c) Ti6Al4V/rhBMP2-loaded hydrogel composite; and the SEM images of cell attachment: (b) bare Ti6Al4V scaffold and (d) Ti6Al4V/rhBMP-2-loaded hydrogel composite. Scale bars in a and c represent $50 \mu \mathrm{m}$; scale bars in b and d represent $100 \mu \mathrm{m}$.

assessments results showed that the Ti6Al4V/rhBMP-2-loaded hydrogel group stimulated the expression of ALP, Runx-2 and COL1 compared with the bare group $(P<0.05)$. Additionally, in both groups, the quantitative assessments showed a significant increase with time from 7 to 14 days.

\subsection{Implantation}

All animals recovered uneventfully after surgery and reached their allocated time point with no infection or postoperative morbidity noted.

3.6.1 Fluorescence analysis. The bone remodeling was validated by the interrupted fluorescent bone markers and new bone deposition. In both groups, the markers were detected in the regenerated bone in the pores of the scaffold (Fig. 6a and b, Tetracycline showed yellow and calcein green showed green). For both the new ingrown bone in pores and the periprosthetic bone, 8 measurements were taken along the span of each double label. Then the averaged thickness of newly mineralized bone was divided by the eleven-day mark interval and expressed in units of microns per day. A significantly greater MAR was measured in the bone ingrowth compared to the periprosthetic bone of both groups at week 1 , weeks 4 and weeks $8(P<0.05)$. Additionally, at each time point, the MAR of the bone ingrowth was significantly greater in the Ti6Al4V/hydrogel group than the bare group $(P<0.05)$ (Fig. 7a). There was no significant difference in the MAR of the periprosthetic bone measured between the two groups at week 1 , weeks 4 and weeks $8(P>0.05)$ (Fig. 7a).
3.6.2 Histomorphometric analysis. Histological observations after HE staining were showed in the Fig. $6 \mathrm{c}$ and $\mathrm{d}$. The bone tissue was in red color while implant was in black color. One week after the implantations, in both groups, a small amount of new bone tissue grew from the edge of bone defect and began to integrate with the periphery of porous implants. A small amount of newly formed bone deposited directly on the surface of the implants and only minor bone ingrowth into the outer region. Four weeks later of implantation, the images showed bone formation progressed at the marginal region of the bone defect into the pores. Bone integration was observed along the implant surface. At week 8, new bone was observed formed toward the center of the defect, which was connected to the bone of the edge of the wound, and the trabecular bone was thicker than at 4 weeks. Much new bone was formed around the implant, and rows of osteoblasts lined around the new bone were observed. Smaller pores were completely filled with bone, whereas larger pores were partially filled. In some parts of the implant, the newly formed bone has successfully bridged the bone defect. The group with rhBMP-2 loaded hydrogel seemed to have more bone-formation than the bare group. The general tissue response was similar for both groups, no osteoclastic reaction was observed in the vicinity of the implant, no material fragments or particles were detected in the surrounding tissue, inflammatory infiltration consisting of mononuclear cells was not observed, nor was infiltration of neutrophils and eosinophils. The general tissue response demonstrates good biocompatibility of the two types of porous implants. The quantitative data of BIC are shown in Fig. 7b. The extent of bone ingrowth 
Bare Ti6Al4V

CCK-8

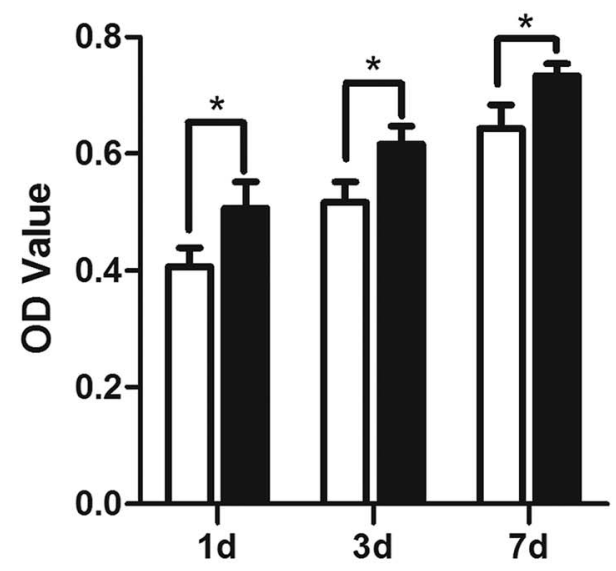

Runx-2

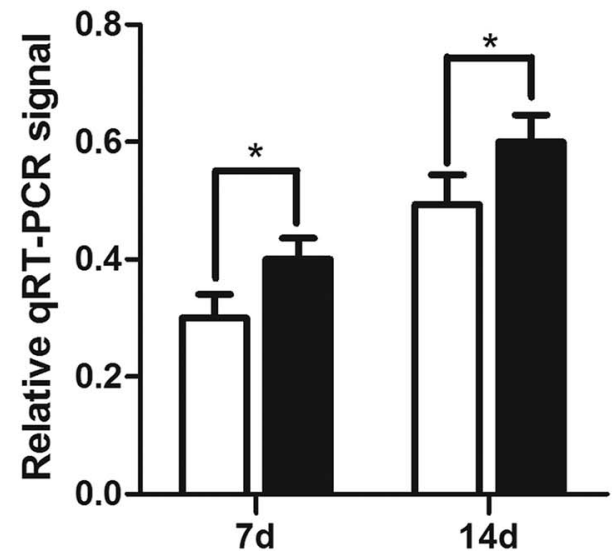

Ti6A14V/Hydrogel

ALP

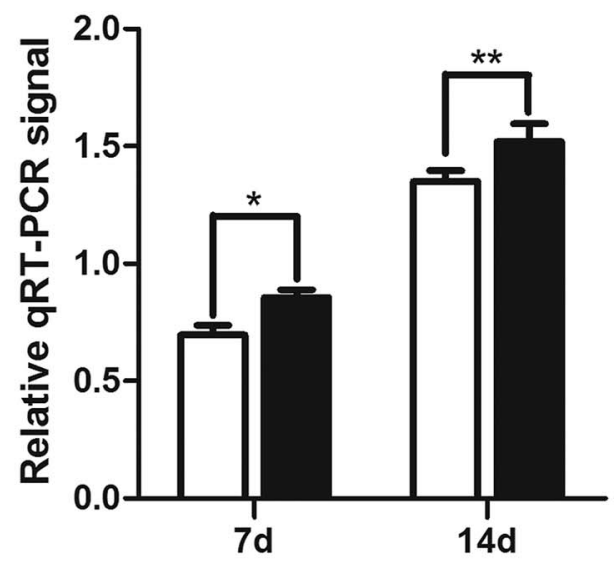

Col I

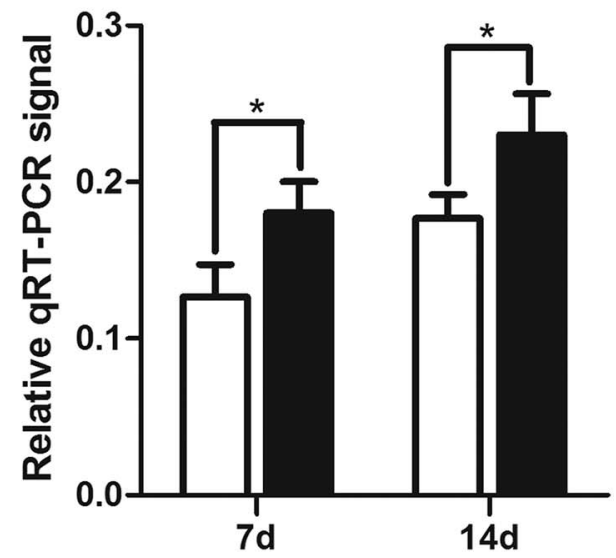

Fig. 5 The CCK-8 results of cell proliferation and the expression of ALP, Runx-2 and Col I in the bare Ti6Al4V group and Ti6Al4V/rhBMP-2loaded hydrogel composite group.

and remodeling onto and inside the porous samples in both groups increased significantly with time. However, the BIC values in the Ti6Al4V/hydrogel group were significantly higher than the bare Ti6Al4V scaffold group at every time point.

\section{Discussion}

Recently, porous Ti6Al4V scaffolds have received increasing attention as a new biomaterial that can act as an osteoconductive scaffold that provides direct mechanical support in large bone defects at weight-bearing sites. ${ }^{27}$ However, many conventional techniques used to produce porous Ti6Al4V led to low porosity and the high possibility of pore blockage, ${ }^{28,29}$ and had very limited control of the internal pore architecture and the external shape of the porous titanium implants. ${ }^{30} \mathrm{~A}$ new powder sintering technique was used in this study, by controlling the quantity and size of spacer particles added to the Ti6Al4V powder, the porous implants could be fabricated with different porosities and pore sizes, thereby the structural design can be fine-tuned so that porous titanium scaffolds offer optimal mechanical and structural conditions required for bone regeneration. ${ }^{31}$ Previous studies have indicated that porous materials with a porosity of $30-90 \%$ and pore size of 150-600 $\mu \mathrm{m}$ can allow bone ingrowth, ${ }^{32,33}$ and higher porosity with larger pores can provide sufficient space for the bone to grow into. ${ }^{34}$ But with the increase in porosity, mechanical properties of the porous material decrease. ${ }^{5}$ Thus, a balance between the two parameters was very important for a better implant performance. In the present study, according to the micro-CT and SEM results, the Ti6Al4V scaffold showed 3D connectivity porous structure. The porosity can reach $74.8 \%$ with pore size about $502 \mu \mathrm{m}$. According to the results of mechanical tests, the scaffold has high compressive strengths (97.5 $\mathrm{MPa})$, and relatively low elastic modulus value (1.83 GPa). ${ }^{35}$ These results were similar to our previous studies, ${ }^{22,23}$ indicating the success fabrication of the porous Ti6Al4V scaffolds. In summary, the porous Ti6Al4V scaffold fabricated by the new sintered technique provides sufficient space for cell and bone to grow into and adequate mechanical support without obvious stress shielding effect.

Previous studies had demonstrated the bone regeneration effect of BMP-2. ${ }^{12}$ However, administering rhBMP-2 in 

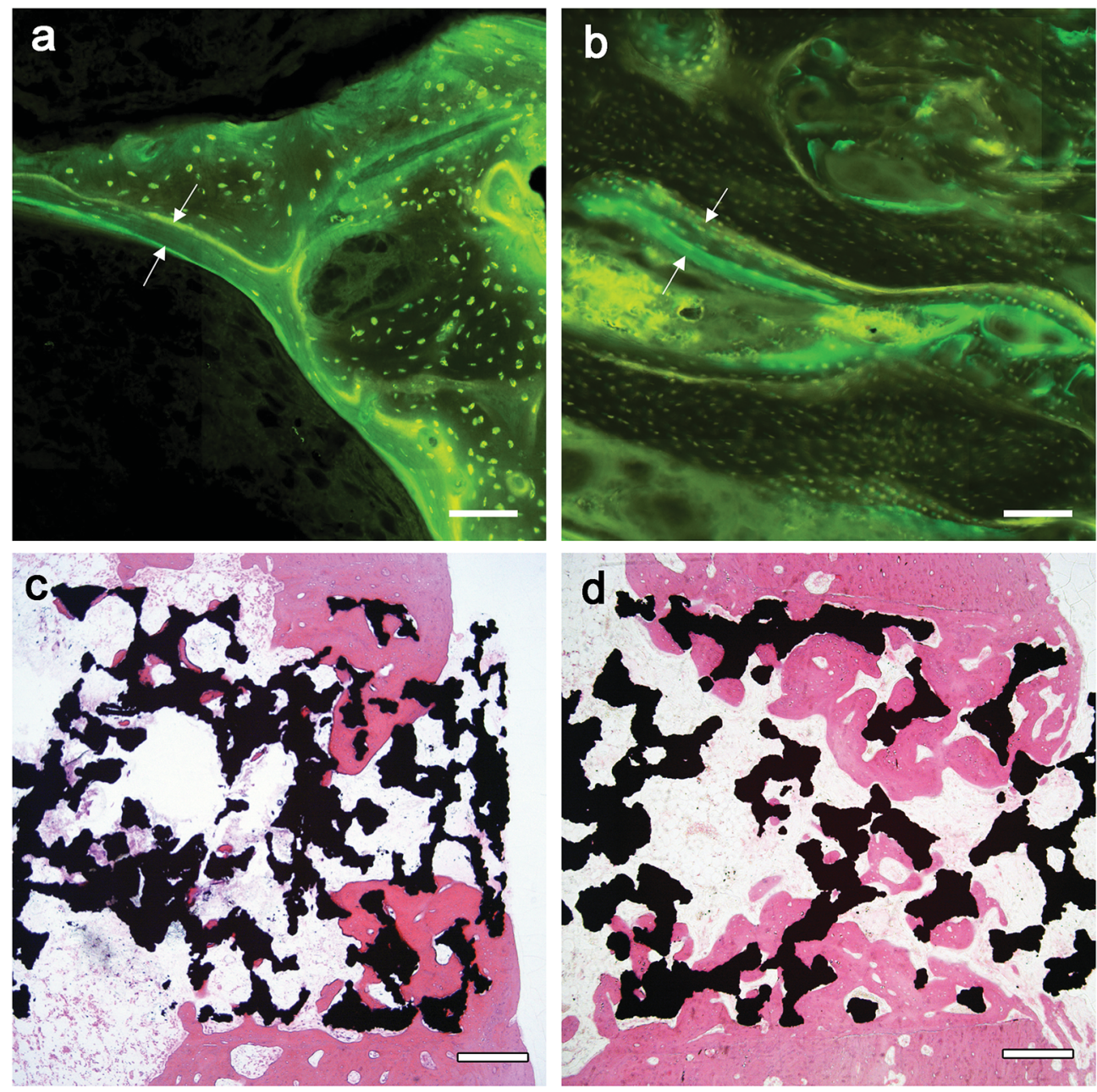

Fig. 6 Fluorescence images of the two groups: (a) bare Ti6Al4V scaffold and (b) Ti6Al4V/rhBMP-2-loaded hydrogel composite. Bone mineralization apposition rate is a vertical space between two fluorochrome markers interval (space between the white arrows). Scale bars represent $30 \mu \mathrm{m}$; histological results after implantation of 4 weeks in the two groups with H\&E staining: (c) bare Ti6Al4V scaffold and (d) Ti6Al4V/rhBMP-2loaded hydrogel composite. Scale bars represent $300 \mu \mathrm{m}$.

orthopedic applications is limited by its short half-life, localized action, and rapid local clearance. ${ }^{36}$ Therefore, it is very important to control the release and prolong the action time of the growth factor. ${ }^{37}$ A number of soft hydrogels such as fibrin, alginate, or collagen groups have been developed recently, which can be used to entrap growth factors, enable a good distribution and a good control of factors. ${ }^{38,39}$ In this study, the thermosensitive CS-TA hydrogel was used to distribute BMP-2 microspheres in low temperature, and then injected into the pores of Ti6Al4V scaffold. The hydrogel changed from mobile fluid to solidified gels after heating in a $37{ }^{\circ} \mathrm{C}$ water bath in $3 \mathrm{~min}$. Rheological properties of the hydrogel and the rhBMP-2 loaded hydrogel were evaluated. The liquid hydrogel could gelled at the temperature of $28.3^{\circ} \mathrm{C}$, and the rhBMP-2 loaded hydrogel was at $29.2{ }^{\circ} \mathrm{C}$. From the curves, we can indicate that the increase of temperature contributed to the gelation, and the thermosensitivity of CS-TA hydrogel and the rhBMP-2 loaded hydrogel were verified. Furthermore, dissolution is an important parameter, which affects the stability of the biomaterial and release of the loaded drug. In the present study, we noted a stable dissolution profile of the rhBMP-2 loaded hydrogel with no sudden increase in the dissolution behavior. Such a controlled dissolution with no burst release is important to avoid pharmacologically dangerous effects. ${ }^{37}$ Therefore, the CSTA hydrogel may be a suitable vehicle for decreasing the burst release of rhBMP-2 microspheres in vivo because of the thermosensity and the high viscosity. However, the hydrogel often lacks the mechanical properties necessary for load-bearing applications. In this study, we combined the rhBMP/hydrogel with the Ti6Al4V scaffolds to obtain enough sufficient mechanical support. In summary, incorporation of hydrogel capable of time- and dose-controlled delivery of rhBMP-2 into porous titanium scaffolds offers a mechanically strong and biologically active composite biomaterial suitable for grafting large bone defects.

In this study, human OB cells were used for the comprehensive cytotoxicity evaluation to simulate the implantation of biomaterials into bone in vivo. ${ }^{40}$ The CCK-8 and live/dead assay were used to study the effect of dissolution of hydrogel infiltrated in Ti6Al4V scaffolds on cell proliferation and viability. 


\section{Bare Ti6Al4V}

a

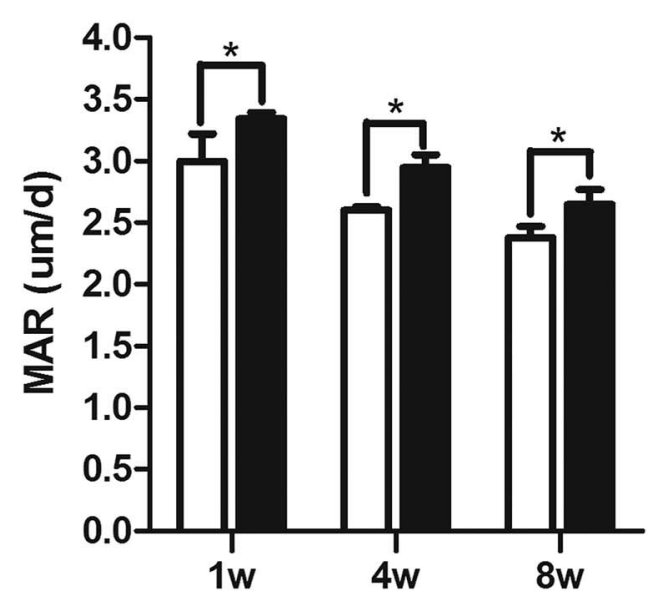

Ti6Al4V/Hydrogel

b

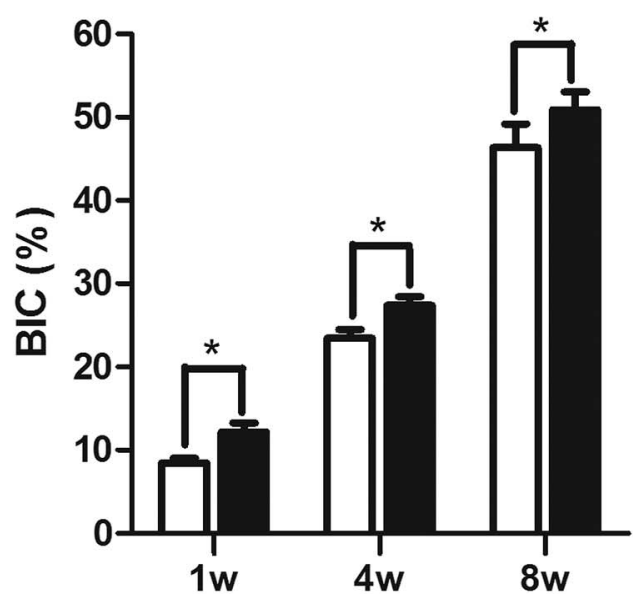

Fig. 7 The values of (a) MAR and (b) BIC in the two groups at 1, 4 and 8 weeks after implantation. *, $P<0.05$. MAR, mineralization apposition rate. BIC, bone-implant contact.

The results showed that cell proliferation on the surface of materials in the Ti6Al4V/hydrogels group was significantly higher than the bare group at each time point. The SEM images showed that cells grew better on the material surface with the presence of rhBMP-2 loaded hydrogel, in which some cells even grew deeply into the pores. These results indicated the highly bioactive and cytocompatible of the Ti6Al4V/hydrogels construct. The expressions of genes and proteins related to bone growth are valuable markers for demonstrating the osteoblast phenotype in vitro. A significantly higher ALP activity was observed with the presence of rhBMP-2 loaded hydrogel when compared to the control samples at each time point. A high value of ALP implies active bone formation because ALP is released as a byproduct of the active osteoblast. ${ }^{41}$ The expression of Runx-2, Col I, BSP and osteocalcin also up-regulated significantly after 7 and 14 days of incubation in both groups. The porous structure supported an enlarged and more suitable environment for bone-like tissue formation and compared with the other group and other researches, and the presence of rhBMP-2 loaded hydrogel extends the lifespan of osteogenic cells and possibly their synthetic activity phase, ultimately leading to an increase in bone tissue formation. ${ }^{42}$

In vivo implantations were performed to identify the in vitro results. Our previous research demonstrated that the sintered porous Ti6Al4V scaffold is endowed with the osteoconductive property which could guide the ingrowth of surrounding bone tissue to form new bone within. ${ }^{22,23}$ In the current work, the Ti6Al4V/hydrogels construct not only preserved the scaffold's osteoconductive property but also further conferred it with osteoinductive potential which is originating from the rhBMP-2 loaded hydrogel. The composite of rhBMP-2 loaded hydrogel filled in the pores of the scaffold is not expected to suppress the cell growth and angiogenesis because of the highly bioactive and porous nature of the hydrogel with interconnected pores. ${ }^{13,43}$ The results of fluorescence and histomorphometric analysis, and micro-CT showed that bone grows from the host bone to the implants, characteristics of direct adhesion of bone to the implant were observed. In both groups, bone ingrowth was observed as early as 1 week postoperatively, the penetration depth of new bone regeneration increased rapidly with the implantation time, indicating good osteoconductivity of the implants. Higher bone growth ratios were found in the scaffold than the periprosthetic bone in both groups. High degrees of BIC were found in both groups, indicating the ability to provide biological anchorage for surrounding bone tissues. The high ingrowth values create a stable ingrowth construct while also maintaining the mechanical strength due to the integration of the porous structure and solid substrate. However, at each point of follow-up, the Ti6Al4V/hydrogels group showed significant faster bone growth speed and more bone ingrowth. The results displayed subsequent positive effect of the rhBMP-2-loaded hydrogels on new bone formation in 8 weeks.

However, there were some limitations of this study. Firstly, the control is not adequate. The group of hydrogel treated porous Ti6Al4V scaffold without rhBMP-2 was absence. Although the promoting osteogenesis of rhBMP-2 have been proven in many previous studies, without that control the effect of rhBMP-2 remains an open question because one cannot assess the proportion of the osteoblast activity enhancement that is due merely to the presence of hydrogel in this study. Secondly, the number of animals and the follow-up period were limited, many adverse effects of the implant may not yet appear. A large number of animals with longer follow-up after implantation may be necessary in the further research to better evaluate the material's biosafety.

\section{Conclusion}

The porous Ti6Al4V/rhBMP-2 loaded hydrogels construct could provide sufficient space and adequate mechanical support 
without obvious stress shielding effect for bone ingrowth, more important, the composite construct showed good biocompatibility and higher osteogenic ability with the presence of rhBMP2 loaded CS-TA hydrogels than the bare scaffold. Therefore, the sintered porous Ti6Al4V scaffolds incorporated with rhBMP-2 and CS-TA hydrogels enhanced the bone regeneration.

\section{Conflicts of interest}

There are no conflicts to declare.

\section{Acknowledgements}

This work was supported by The National Key Research and Development Program (grant numbers: 2016YFC1101905).

\section{References}

1 C. M. Court-Brown and M. M. Mcqueen, J. Bone Jt. Surg., Am. Vol., 2016, 98, e36.

2 M. J. Yaszemski, R. G. Payne, W. C. Hayes, R. Langer and A. G. Mikos, Biomaterials, 1996, 17, 175.

3 D. I. Ilan and A. L. Ladd, Operative Techniques in Plastic \& Reconstructive Surgery, 2012, vol. 28, pp. 457-468.

4 G. Lewis, J. Mater. Sci.: Mater. Med., 2013, 24, 2293-2325.

5 J. Parthasarathy, B. Starly, S. Raman and A. Christensen, J. Mech. Behav. Biomed. Mater., 2010, 3, 249-259.

6 P. Heinl, L. Müller, C. Körner, R. F. Singer and F. A. Müller, Acta Biomater., 2008, 4, 1536-1544.

7 d. S. J. Van, H. Wang, Y. S. Amin, M. Siebelt, M. Sandker, J. H. Waarsing, J. A. Verhaar, H. Jahr, A. A. Zadpoor and S. C. Leeuwenburgh, Tissue Eng., Part A, 2013, 19, 2605-2614. 8 A. Kumar, S. Mandal, S. Barui, R. Vasireddi, U. Gbureck, M. Gelinsky and B. Basu, Mater. Sci. Eng., R, 2016, 103, 1-39.

9 K. Rezwan, Q. J. Chen and A. R. Boccaccini, Biomaterials, 2006, 27, 3413-3431.

10 J. J. de Obarrio, J. I. Araãoz-Dutari, T. M. Chamberlain and A. Croston, Int. J. Periodontics Restorative Dent., 2000, 20, 486-497.

11 K. Takaoka, H. Yoshikawa, J. Hashimoto, S. Miyamoto, K. Masuhara, H. Nakahara, M. Matsui and K. Ono, Clinical orthopaedics and related research, 1993, pp. 329-336.

12 D. H. Kempen, L. Lu, T. E. Hefferan, L. B. Creemers, A. Maran, K. L. Classic, W. J. Dhert and M. J. Yaszemski, Biomaterials, 2008, 29, 3245-3252.

13 M. Vishnu Priya, A. Sivshanmugam, A. R. Boccaccini, O. M. Goudouri, W. Sun, N. Hwang, S. Deepthi, S. V. Nair and R. Jayakumar, Biomed. Mater., 2016, 11, 035017.

14 J. W. McClellan, D. S. Mulconrey, R. J. Forbes and N. Fullmer, J. Spinal Disord. Tech., 2006, 19, 483-486.

15 K. Takaoka, M. Koezuka and H. Nakahara, J. Orthop. Res., 2010, 9, 902.

16 Q. Zhang, Orthopaedic Biomechanics Materials \& Clinical Study, 2005.

17 V. Luginbuehl, L. Meinel, H. P. Merkle and B. Gander, Eur. J. Pharm. Biopharm., 2004, 58, 197-208.
18 Y. Fu, L. Du, Q. Wang, W. Liao, Y. Jin, A. Dong, C. Chen and Z. Li, Pharmazie, 2012, 67, 299-303.

19 E. J. Carragee, E. L. Hurwitz and B. K. Weiner, Spine J., 2011, 11, 471-491.

20 Y. Fu, Q. Zhang, Y. Sun, W. Liao, X. Bai, L. Zhang, L. Du, Y. Jin, Q. Wang, Z. Li and Y. Wang, J. Orthop. Res., 2014, 32, 744-751.

21 S. Mullens, I. Thijs, J. Cooymans and J. Luyten, US Pat. US8992828, 2015.

22 J. Li, Z. Li, Y. Shi, H. Wang, R. Li, J. Tu and G. Jin, J. Mech. Behav. Biomed. Mater., 2018, 91, 149-158.

23 J. Li, Z. L. Li, R. L. Li, Y. Y. Shi, H. R. Wang, Y. X. Wang and G. Jin, RSC Adv., 2018, 8, 36512-36520.

24 J. B. Oldham, L. Lu, X. Zhu, B. D. Porter, T. E. Hefferan, D. R. Larson, B. L. Currier, A. G. Mikos and M. J. Yaszemski, J. Biomech. Eng., 2000, 122, 289-292.

25 J. H. Park, M. Ye and K. Park, Molecules, 2005, 10, 146-161. 26 A. Hatefi and B. Amsden, J. Control. Release, 2002, 80, 9-28. 27 M. Niinomi and M. Nakai, Int. J. Biomater., 2011, 2011, 836587.

28 K. Korkmaz, Surf. Coat. Technol., 2015, 272, 72-78.

29 J. Ruan, H. Yang, X. Weng, J. Miao and K. Zhou, J. Mater. Sci. Mater. Med., 2016, 27, 1-8.

30 G. Ryan, A. Pandit and D. P. Apatsidis, Biomaterials, 2006, 27, 2651-2670.

31 J. Van der Stok, O. P. Van der Jagt, S. Amin Yavari, M. F. De Haas, J. H. Waarsing, H. Jahr, E. M. Van Lieshout, P. Patka, J. A. Verhaar, A. A. Zadpoor and H. Weinans, J. Orthop. Res., 2013, 31, 792-799.

32 W. Mróz, B. Budner, R. Syroka, K. Niedzielski, G. Golański, A. Slósarczyk, D. Schwarze and T. E. Douglas, J. Biomed. Mater. Res. B, 2015, 103, 151-158.

33 Y. S. Amin, d. S. J. Van, Y. C. Chai, R. Wauthle, B. Z. Tahmasebi, P. Habibovic, M. Mulier, J. Schrooten, H. Weinans and A. A. Zadpoor, Biomaterials, 2014, 35, 6172-6181.

34 D. Muller, H. Chim, A. Bader, M. Whiteman and J. T. Schantz, Stem Cells Int., 2010, 2011, 547247.

35 A. Syahrom, M. R. Abdul Kadir, J. Abdullah and A. Ö, Medical Engineering \& Physics, 2013, 35, 792-799.

36 F. Kenji, M. Yukihide, I. Kentaro, I. Hiroyoshi, N. Hiroaki and T. Kunio, J. Arthroplasty, 2012, 27, 143-149.e141.

37 S. Cascone, Eur. J. Pharm. Sci., 2017, 106, 352-361.

38 J. L. Drury and D. J. Mooney, Biomaterials, 2003, 24, 43374351.

39 A. Mellati, C. M. Fan, A. Tamayol, N. Annabi, S. Dai, J. Bi, B. Jin, C. Xian, A. Khademhosseini and H. Zhang, Biotechnol. Bioeng., 2017, 114, 217-231.

40 S. Minagar, J. Wang, C. C. Berndt, E. P. Ivanova and C. Wen, J. Biomed. Mater. Res., Part A, 2013, 101A, 2726-2739.

41 A. Sabokbar, P. J. Millett, B. Myer and N. Rushton, Bone Miner., 1994, 27, 57-67.

42 E. M. Lotz, R. Olivares-Navarrete, S. Berner, B. D. Boyan and Z. Schwartz, J. Biomed. Mater. Res., Part A, 2016, 104, 31373148.

43 O. Oliviero, M. Ventre and P. A. Netti, Acta Biomater., 2012, 8, 3294-3301. 